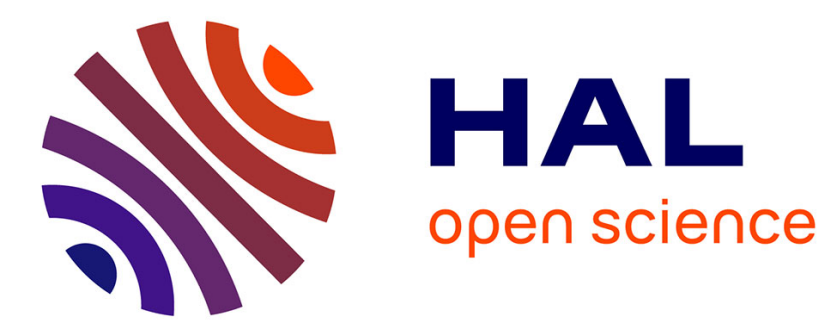

\title{
On-Line Fault Diagnosis of Dynamic Systems via Robust Parameter Identification
}

Gérard Bloch, Mustapha Ouladsine, Philippe Thomas

\section{To cite this version:}

Gérard Bloch, Mustapha Ouladsine, Philippe Thomas. On-Line Fault Diagnosis of Dynamic Systems via Robust Parameter Identification. Control Engineering Practice, 1995, 3 (12), pp.1709-1717. 10.1016/0967-0661(95)00183-U . hal-00867377

\section{HAL Id: hal-00867377 https://hal.science/hal-00867377}

Submitted on 29 Sep 2013

HAL is a multi-disciplinary open access archive for the deposit and dissemination of scientific research documents, whether they are published or not. The documents may come from teaching and research institutions in France or abroad, or from public or private research centers.
L'archive ouverte pluridisciplinaire HAL, est destinée au dépôt et à la diffusion de documents scientifiques de niveau recherche, publiés ou non, émanant des établissements d'enseignement et de recherche français ou étrangers, des laboratoires publics ou privés. 


\title{
On-Line Fault Diagnosis of Dynamic Systems via Robust Parameter Identification
}

\author{
Gérard Bloch $^{\mathrm{a}}$, Mustapha Ouladsine ${ }^{\mathrm{a}}$, Philippe Thomas ${ }^{\mathrm{a}}$ \\ ${ }^{a}$ Centre de Recherche en Automatique de Nancy, CNRS URA D821 ESSTIN, Rue Jean Lamour, 54500 Vandoeuvre, France
}

\begin{abstract}
A procedure simultaneously achieving the detection of faults, their isolation and their identification is presented. The systems considered are MISO systems represented by ARX models, the parameters of which are estimated on-line by a robust procedure. A priori knowledge of the faults which can occur is used. The faults modeled here are outliers, biases or drifts, and can act upon output, inputs or even noise. The magnitude of a fault is estimated on a moving window from the prediction error sequence by least squares. Statistical tests of the significance of the estimated parameters corresponding to the different faults are performed. An application on the strip drive in the furnace of an annealing line is finally presented.
\end{abstract}

Key words: fault detection, fault isolation, fault identification, analytical redundancy, robust recursive identification.

\section{Introduction}

Numerous and various factors motivate the integration of fault detection and isolation (FDI) methods in the supervision systems of industrial plants: the growing complexity of these plants and their control systems, the development of the "just in time" production, which calls for increasing operational availability, total quality policies, importance attached to the risks, etc. This growing demand for fault tolerance can be achieved by improving the individual reliability of the different pieces of equipment, and by developing systematic preventive maintenance strategies. Another complementary way is system state monitoring in real time. Although restricted in the past to domains with a high risk level, such as aeronautics and astronautics, or nuclear power plants, the diagnostic function tends to be present in all production fields.

Generally speaking, diagnosis can be viewed as a complex sequential procedure involving three steps: detection of the operating modes, qualification of these modes (identification and localization of the cause), and decision. Although several classifications can be applied, the diagnostic methods can be presented in three classes exploiting respectively physical redundancy (the use of repeated identical components), functional (analytical) models, or heuristic knowledge expressed, for example, in the form of rules, trees or qualitative models. This paper is concerned only with the second approach, namely the model-based approach.

Fault detection and isolation (FDI) using analytical redundancy methods are currently the subject of extensive research and numerous surveys can be found. See for instance Willsky (1976), Isermann (1984), Basseville (1988), Frank (1990), Patton and Chen (1991). More recently, a review and a comparison of the different FDI approaches has been made by Isermann (1994), who proposes a framework for integrating analytical symptoms (given from functional models) and heuristic symptoms. The model-based approaches use either signal models leading to spectral analysis (Neumann, 1991) and parameter estimation of ARMA models, or process models leading to parameter estimation (Gomm et al., 1993), state estimation or use of parity equations. The performances of the parity space and parameter estimation approaches for the detection of additive and multiplicative faults have been compared by Höfling and Pfeufer (1994). Methods based on a state estimation using Kalman filters or observers rely on the use of accurate models, and on numerous assumptions such as known model structure and parameters, known noise characteristics and known effects of the faults on the model. Robustness issues on model uncertainties are at present being studied (Patton, 1994).

Although several studies are based on the representation of systems by transfer functions (Mironovski, 1980), most of the time the methods are based on a state representation of systems, and assume that the nature of the possible faults is not previously known. Therefore the different steps are clearly separated: detection, isolation and, if possible, estimation of the magnitude of the fault.

In contrast, a procedure is presented here which simultaneously achieves the detection of faults, their localization and their identification. The main idea is to make intensive use of the knowledge concerning the faults assumed to occur in the system. The procedure can be related to the intervention analysis, provided by Box and Tiao (1975) for time series modeling, and to further works aiming at handling outliers in identification (Tsay, 1988) (Kobi et al., 1993a,b). Chen and Patton (1994) emphasize too the importance of modeling systems with sensor, actuator and component faults in the fault detectability and isolability problems.

In order to detect, locate and identify the faults, the model of the system being considered must be known. Nevertheless, the case of unknown model parameters is in fact the most frequent. Thus, the parameters of the model must be identified on-line, by a recursive procedure insensitive to the events to be detected. A robust identification algorithm, developed by Puthenpura and 
Sinha (1990), is then used to provide the procedure for the detection, localization and identification of the faults with the parameters of the model.

In the second part of this paper, systems are modeled by decomposition into multi-input/single-output ARX models. Such models can often be considered as a good compromise between simplicity and power of description. As pointed out by Gomm et al. (1993), they have the main advantage that they are obtained by experimental, and not theoretical, analysis and are therefore applicable to a wide range of processes. Models of the different faults, taking into account their dynamics and their application points (locations), are built and then reduced to a unique form. The faults considered are outliers, biases or drifts concerning both sensors or actuators of the monitored system. These types of faults represent the majority of the faults which can be encountered in practice, apart from specific problems related to particular systems.

In the third part, the procedure of fault detection, isolation and estimation is presented. Different assumptions are considered. With the first one, the type, location and occurrence time of the fault and system model parameters are assumed to be known, and the estimation of the fault magnitude is performed simply by least squares. In the second method, only the time of the fault occurrence and the system model parameters are known, and statistical tests of the significance of the estimated parameters corresponding to the different faults are performed. In the third place, the time of the fault occurrence is also unknown, and the previous estimations and tests are carried out on a moving window. Finally, the parameters of the model of the system are assumed to be unknown and are identified on-line, allowing the complete diagnostic procedure to be performed, which can be implemented in real time.

The last section is devoted to an example dealing with the strip drive in the furnace of an annealing line.

\section{The models}

The MIMO systems which can be decomposed into $n_{y}$ MISO subsystems, where $n_{y}$ is the number of outputs, are considered. Each MISO subsystem is represented by an ARX model in discrete-time $t$ :

$$
A(q) y(t)=\sum_{j=1}^{p} B_{j}(q) u_{j}\left(t-n d_{j}\right)+\varepsilon(t)
$$

where $A(q)=1+a_{1} q^{-1}+\cdots+a_{n a} q^{-n a}$, $B_{j}(q)=b_{j 1}+b_{j 2} q^{-1}+\cdots+b_{j n b_{j}} q^{-n b_{j}+1}, j=1, \ldots, p$, are polynomials in the delay operator $q^{-1}$, such as $q^{-1} y(t)=y(t-1)$, of orders $n a$ and $n b_{j}, j=1, \ldots, p$, respectively, $y(t)$ is the output of the system, $u_{j}(t), j=1, \ldots, p$, are the $p$ inputs of the systems, $n d_{j}, j=1, \ldots, p$, are the delays from inputs to output and $\{\varepsilon(t)\}$ is a sequence of independent and normally distributed variables (white noise) with mean zero and variance $\sigma_{e}^{2}$ when there is no fault. In model (1), it is assumed that all of the zeros of the polynomials are outside the unit circle.
When various disturbances act on the inputs and the output, they are unobservable, and the observed variables can be written as:

$$
\begin{gathered}
Y(t)=y(t)+f_{y}(t), \\
U_{j}(t)=u_{j}(t)+f_{u_{j}}(t), j=1, \ldots, p,
\end{gathered}
$$

where $f_{y}(t)$ and $f_{u_{j}}(t), j=1, \ldots, p$, are parametric functions representing exogenous deterministic disturbances, for instance measurement errors, such as outliers or biases. Note that for the inputs, $f_{u_{j}}(t)$ can also represent, apart from the sign, a disturbance of the actuator. The real input $u_{j}(t)$ of the system is then linked to the measured input $U_{j}(t)$ by:

$$
u_{j}(t)=U_{j}(t)-f_{u_{j}}(t) .
$$

A change in the system itself can to some extent be represented by a change of the noise $\varepsilon(t)$, expressed as:

$$
E(t)=\varepsilon(t)+f_{e}(t)
$$

The function $f_{e}(t)$ can be deterministic, or stochastic as presented by Tsay (1988), leading to tests of variance change. Only the deterministic case is considered here.

The disturbed system can be represented by the diagram in Figure 1. Whatever its point of application (location) may be, on

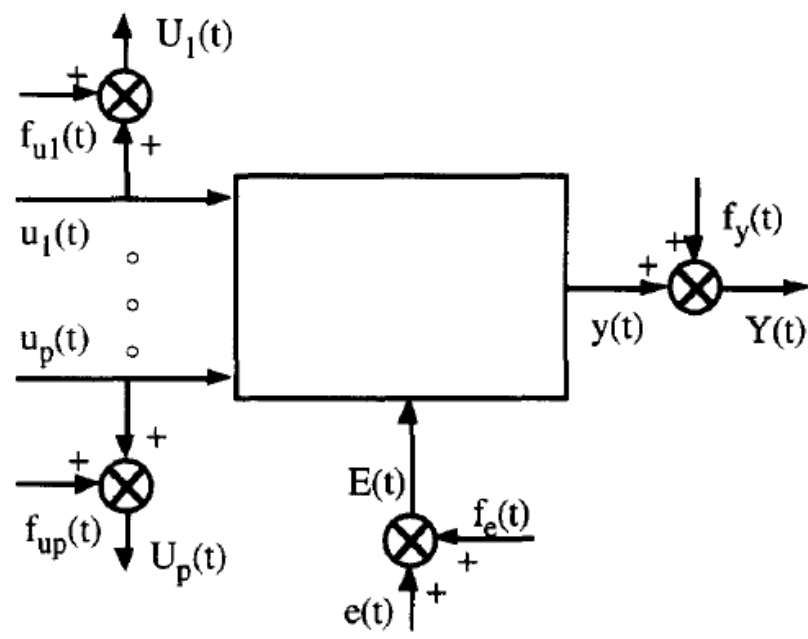

Figure 1: The system and its disturbances

the output, on the inputs or on the equation noise, it is assumed that the disturbance function (respectively $f_{y}(t), f_{u_{j}}(t)$ and $f_{e}(t)$ ), noted simply $f(t)$, can be written as:

$$
f(t)=\omega \frac{\Omega_{D}(q)}{\Delta_{D}(q)} \xi_{t}^{d}=\omega P_{D}(q) \xi_{t}^{d},
$$

where $\xi_{t}^{d}$ is an indicator variable signifying the occurrence of a disturbance at the time point $d\left(\xi_{t}^{d}=1\right.$ if $t=d, \xi_{t}^{d}=0$ if $\left.t \neq d\right)$. $\Omega_{D}(q)$ and $\Delta_{D}(q)$ are polynomials representing the dynamics of the disturbance and $\omega$ is a constant denoting its initial impact. The main disturbance shapes which can be met in practice are the following. If $P_{D}(q)=1, f(t)$ represents an outlier of magnitude $\omega$, at time $d$. If $P_{D}(q)=\frac{1}{1-q^{-1}}, f(t)$ represents a level 
change of magnitude $\omega$, at time $d$, and renders a bias of the corresponding sensor of magnitude $\omega$. If $P_{D}(q)=\frac{1}{\left(1-q^{-1}\right)^{2}}, f(t)$ represents a drift of slope $\omega$ from instant $d$.

Using equation (1), the prediction error $e(t)$ can be calculated from the measurements assumed without faults as:

$$
e(t)=A(q) y(t)-\sum_{j=1}^{p} B_{j}(q) u_{j}\left(t-n d_{j}\right)
$$

A variable $z(t)$ can also be built from the actual measurements:

$$
z(t)=A(q) y(t)-\sum_{j=1}^{p} B_{j}(q) U_{j}\left(t-n d_{j}\right)
$$

If a fault occurs on $y(t)$ only, using (2), the previous equation becomes:

$$
z(t)=A(q) y(t)-\sum_{j=1}^{p} B_{j}(q) u_{j}\left(t-n d_{j}\right)+A(q) f_{y}(t) .
$$

If a fault occurs on $u_{k}(t)$ only, $z(t)$ now becomes, using (3):

$$
z(t)=A(q) y(t)-\sum_{j=1}^{p} B_{j}(q) u_{j}\left(t-n d_{j}\right)+B_{k}(q) f_{u_{k}}(t) .
$$

Moreover if a change occurs on the noise $\varepsilon(t)$, one obtains by (4) simply:

$$
z(t)=A(q) Y(t)-\sum_{j=1}^{p} B_{j}(q) u_{j}\left(t-n d_{j}\right)+f_{e}(t) .
$$

The three preceding versions of equation (7) can be reduced to a unique form and combined with (6):

$$
z(t)=e(t)+F(t)
$$

where

$$
F(t)=P_{L}(q) f(t)=P_{L}(q) P_{D}(q) \xi_{t}^{d} \omega
$$

and where $P_{L}(q)=A(q), P_{L}(q)=-B_{k}(q)$ or $P_{L}(q)=1$ if a fault or a change occurs on the output $y(t)$, on the kth input $u_{k}(t)$ or on the noise $\varepsilon(t)$, respectively.

\section{The detection-isolation-estimation procedure}

For a better understanding, the complete procedure will be presented in several steps, where more and more characteristics of the disturbance must be determined and fewer and fewer hypotheses are used.

\subsection{Estimation of the magnitude of the disturbance}

Firstly, the disturbance magnitude $\omega$ is estimated using the following hypothesis.

Assumption 1. Fault occurrence instant d, fault type, fault location, structure and parameters of the model are known.
For $n$ time points, the previous equation (8) can be expressed as:

$$
\underline{Z}=\underline{H} \omega+\underline{E},
$$

where

$$
\begin{aligned}
\underline{Z} & =\left[\begin{array}{lll}
z(1) & \ldots & z(d-1) z(d) \ldots z(n)
\end{array}\right]^{T}, \\
\underline{H} & =\left[\begin{array}{llll}
0 & \ldots & 0 & h(d) \ldots h(n)
\end{array}\right]^{T}, \\
\underline{E} & =\left[\begin{array}{lll}
e(1) \ldots e(d-1) e(d) \ldots e(n)
\end{array}\right]^{T},
\end{aligned}
$$

and $\underline{H}$ is the response to an impulse occurring at time $d$ of the transfer function $P_{L}(q) P_{D}(q)$, where $P_{L}(q)$ and $P_{D}(q)$ are polynomials corresponding respectively to the location and the type of fault.

With these notations, the magnitude of the disturbance is simply given by least-squares estimation:

$$
\hat{\omega}=\left(\underline{H}^{T} \underline{H}\right)^{-1} \underline{H}^{T} \underline{Z} .
$$

\subsection{Estimation of the type, location and magnitude of the dis- turbance}

Secondly, the type, location and magnitude of the disturbance are estimated using the following hypothesis.

Assumption 2. The fault occurrence instant d, and the structure and parameters of the model are known.

In this case, $N$ vectors $\underline{H}_{i}, i=1, \ldots, N$, can be built, each one representing a particular disturbance. Therefore $N=n_{L} n_{D}$, where $n_{L}$ is the number of possible application points $\left(n_{L}=\right.$ $p+2$ ) and $n_{D}$ is the number of possible shapes of the fault. The magnitude $\omega_{i}$ corresponding to each type of disturbance can be estimated by:

$$
\hat{\omega}_{i}=\left(\underline{H}_{i}^{T} \underline{H}_{i}\right)^{-1} \underline{H}_{i}^{T} \underline{Z}, i=1, \ldots, N .
$$

The localisation and determination of the disturbance dynamics can be achieved by classical tests related to the linear model. The following variable can, for example, be calculated:

$$
t_{i}=\frac{\hat{\omega}_{i}}{\hat{\sigma}_{i}}, i=1, \ldots, N
$$

which, without fault, i.e. under the hypothesis of nullity of the fault magnitude $\omega_{i}$, follows a Student distribution with $n-1$ degrees of freedom, with the classical hypotheses of normality for vector $\underline{E} . \hat{\sigma}_{i}$ is the standard deviation of the coefficient $\hat{\omega}_{i}$ given by:

$$
\hat{\sigma}_{i}^{2}=\frac{\left(\underline{Z}-\underline{H}_{i} \hat{\omega}_{i}\right)^{T}\left(\underline{Z}-\underline{H}_{i} \hat{\omega}_{i}\right)}{n-1}\left(\underline{H}_{i}^{T} \underline{H}_{i}\right)^{-1} .
$$

Without faults, for each possible fault, i.e. for $i=1, \ldots, N$ :

$$
\left|t_{i}\right|<t_{1-\alpha / 2}
$$

where $t_{1-\alpha / 2}$ is the value of a Student variable with $n-1$ degrees of freedom corresponding to a distribution function equal to $1-$ $\alpha / 2$, with $\alpha$ the risk, i.e. the probability of a type I error, fixed a priori. The index $i_{M}=\arg \max _{i}\left(\left|t_{i}\right||| t_{i} \mid \geq t_{1-\alpha / 2}\right)$ provides both the location and the type of the fault. The corresponding coefficient $\hat{\omega}_{i_{M}}$ gives its magnitude. 


\subsection{Estimation of the type, location, magnitude and occur- rence time of the disturbance}

Thirdly, the type, location, magnitude and occurrence time of the disturbance are estimated using the following hypothesis.

Assumption 3. The structure and the parameters of the model are known.

For on-line operation, the determination of the occurrence time can be made by processing on a moving window. At each time point $t$, i.e. on each moving window of $n=2 m+1$ points, a vector $\underline{Z}(t)=[z(t-m) \ldots z(t-1) z(t) z(t+1) \ldots z(t+m)]^{T}$ is formed, and fault magnitudes $\hat{\omega}_{i}(t), i=1, \ldots, N$, are calculated from vectors $\underline{Z}(t)$ and $\underline{H}_{i}=[0 \ldots 0 h(d=m+1) h(m+2) \ldots h(2 m+1)]^{T}$ corresponding to the different faults:

$$
\hat{\omega}_{i}(t)=\left(\underline{H}_{i}^{T} \underline{H}_{i}\right)^{-1} \underline{H}_{i}^{T} \underline{Z}(t), i=1, \ldots, N .
$$

Then $\hat{\sigma}_{i}^{2}(t)$ and $t_{i}(t), i=1, \ldots, N$, are calculated by:

$$
\begin{gathered}
\hat{\sigma}_{i}^{2}(t)=\frac{\left(\underline{Z}(t)-\underline{H}_{i} \hat{\omega}_{i}(t)\right)^{T}\left(\underline{Z}(t)-\underline{H}_{i} \hat{\omega}_{i}(t)\right)}{n-1}\left(\underline{H}_{i}^{T} \underline{H}_{i}\right)^{-1}, \\
t_{i}(t)=\frac{\hat{\omega}_{i}(t)}{\hat{\sigma}_{i}(t)} .
\end{gathered}
$$

Moreover, the maximum of $\left|t_{i}(t)\right|, i=1, \ldots, N$, is calculated and compared to a threshold, allowing the detection at time $t$. Final localisation and identification are achieved by considering the $t_{i}(t), i=1, \ldots, N$, for the next $2 m+1$ points after the detection point $t_{D}$. The indexes $i_{M}$ and $t_{M}$ for which $\max _{i=1, \ldots, N, t=t_{D}, \ldots, t_{D+2 m}}\left|t_{i}(t)\right|$ is reached give the location and the type of the fault (for $i_{M}$ ) and its occurrence time (for $t_{M}$ ). Its magnitude is given by $\hat{\omega}_{i_{M}}\left(t_{M}\right)$.

\subsection{Complete procedure}

Finally, the type, location, magnitude, occurrence time of the disturbance and the parameters of the model are estimated on line.

\section{Assumption 4. The structure of the model is known.}

The previous procedure uses the model of the system. If the parameters of model (1) are unknown, they can be estimated recursively. However, the disturbance which acts upon the measured variables must not influence the parameter identification and so a robust procedure must be carried out.

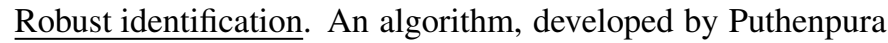
and Sinha (1990), is used in the sequel. This scheme is a weighted least-squares algorithm with particular weights, and is very similar to the robust Kalman filter obtained by Masreliez and Martin (1977).

The identification algorithm is based on the modelling of outliers of Huber (1964), and considers a system represented by the following regression model:

$$
y(t)=\varphi^{T}(t) \theta+\varepsilon(t)
$$

where $y(t)$ is the system output, $\varphi(t)$ is the regression vector, $\theta$ is the true parameter vector and where the equation noise $\varepsilon(t)$ can be expressed as:

$$
\varepsilon(t) \sim(1-\mu) \mathcal{N}\left(0, \sigma_{1}^{2}\right)+\mu \mathcal{N}\left(0, \sigma_{2}^{2}\right),
$$

where $\mathcal{N}\left(0, \sigma^{2}\right)$ represents a normal distribution with mean zero and variance $\sigma^{2} . \mu$ is the probability that large errors occur $(0 \leq \mu \leq 1)$. Note that $\sigma_{2}^{2}>\sigma_{1}^{2}$ and that $\mathcal{N}\left(0, \sigma_{1}^{2}\right)$ describes the basic regular distribution of the noise and $\mathcal{N}\left(0, \sigma_{2}^{2}\right)$ the effect of large errors.

For the identification of the parameter vector $\theta$, model (9) becomes:

$$
y(t)=\varphi^{T}(t) \hat{\theta}+e(t),
$$

where $e(t)$ is the prediction error. The probability $\mu$ being unknown, the model (10) is replaced by:

$$
\varepsilon(t) \sim[1-\delta(t)] \mathcal{N}\left(0, \sigma_{1}^{2}\right)+\delta(t) \mathcal{N}\left(0, \sigma_{2}^{2}\right),
$$

where $\delta(t)=0$ for $|e(t)| \leq M$ and $\delta(t)=1$ for $|e(t)|>M$, and $M$ is a preassigned bound which can be taken as $3 \sigma_{1}$ (Aström, 1980). So the parameter vector $\theta$ is recursively estimated by:

$\hat{\theta}(t)=\hat{\theta}(t-1)+\frac{P(t-1) \varphi(t) e(t)}{\left[(1-\delta(t)) \sigma_{1}^{2}(t)+\delta(t) \sigma_{2}^{2}(t)\right]+\varphi^{T}(t) P(t-1) \varphi(t)}$,

where

$P(t)=P(t-1)-\frac{P(t-1) \varphi(t) \varphi(t)^{T} P(t-1)}{\left[(1-\delta(t)) \sigma_{1}^{2}(t)+\delta(t) \sigma_{2}^{2}(t)\right]+\varphi^{T}(t) P(t-1) \varphi(t)}$.

Note that the variances $\sigma_{1}^{2}(t)$ and $\sigma_{2}^{2}(t)$ are updated by:

$\sigma_{1}^{2}(t)=\sigma_{1}^{2}(t-1)+\frac{1}{t-\tau}\left(e^{2}(t)-\sigma_{1}^{2}(t-1)\right)$, for $|e(t)| \leq 3 \sigma_{1}(t-1)$,

$\sigma_{1}^{2}(t)=\sigma_{1}^{2}(t-1) \quad$, otherwise,

$\sigma_{2}^{2}(t)=\sigma_{2}^{2}(t-1)+\frac{1}{\tau}\left(e^{2}(t)-\sigma_{2}^{2}(t-1)\right), \quad$ for $|e(t)|>3 \sigma_{1}(t-1)$, $\sigma_{2}^{2}(t)=\sigma_{2}^{2}(t-1) \quad$, otherwise,

with $\tau=0$ for $t=1$, and $\tau=\tau+1$ whenever $|e(t)|>3 \sigma_{1}(t-1)$. $\sigma_{2}(0)$ can be chosen as $\sigma_{2}(0)=3 \sigma_{1}(0)$.

Algorithm architecture. Model (1) is expressed in the form (9), where the vector $\theta$ is constituted of the parameters of the polynomials $A(q), B_{j}(q), j=1, \ldots, p$. $\hat{\theta}(t)$ is estimated by the robust recursive procedure (eq. 12 and 13). So the vectors $\underline{H}_{i}(t)=\left[0 \ldots 0 h_{i}(m+1) h_{i}(m+2) \ldots h_{i}(2 m+1)\right]^{T}(t)$ are calculated for $i=1, \ldots, N=n_{L} n_{D}$, where $n_{L}$ and $n_{D}$ are respectively the numbers of possible application points and shapes of the faults. Each vector $H_{i}(t)$ corresponds to the response to an impulse occurring at time $m+1$ of the transfer function $\hat{P}_{L, j}(q) P_{D, k}(q), j=1, \ldots, n_{L}, k=1, \ldots, n_{D}$. Note that $\hat{P}_{L, j}(q)$ is obtained from the parameter set $\hat{\theta}(t)$ estimated at time $t$.

Then, for each moving window of $n=2 m+1$ points, the vector 
$\underline{Z}(t)=[z(t-m) \ldots z(t-1) z(t) z(t+1) \ldots z(t+m)]^{T}$ is formed and the following quantities are calculated for $i=1, \ldots, N$ :

$$
\begin{gathered}
\hat{\omega}_{i}(t)=\left(\underline{H}_{i}^{T}(t) \underline{H}_{i}(t)\right)^{-1} \underline{H}_{i}^{T}(t) \underline{Z}(t), \\
\hat{\sigma}_{i}^{2}(t)=\frac{\left(\underline{Z}(t)-\underline{H}_{i}(t) \hat{\omega}_{i}(t)\right)^{T}\left(\underline{Z}(t)-\underline{H}_{i}(t) \hat{\omega}_{i}(t)\right)}{n-1}\left(\underline{H}_{i}^{T}(t) \underline{H}_{i}(t)\right)^{-1}, \\
t_{i}(t)=\frac{\hat{\omega}_{i}(t)}{\hat{\sigma}_{i}(t)} .
\end{gathered}
$$

The maximum of $\left|t_{i}(t)\right|, i=1, \ldots, N$, is calculated and compared to a threshold, allowing detection at time $t$. Note that the measurements until time $t+m$ have been used to form $\underline{Z}(t)$, introducing a detection delay of $m$ points. The final isolation and identification are achieved by considering the $t_{i}(t), i=1, \ldots, N$, for the next $n=2 m+1$ points after the detection point $t_{D}$. The indexes $i_{M}$ and $t_{M}$ for which $\max \quad\left|t_{i}(t)\right|$ is reached give the location and type of the fault (for $i_{M}$ ) and its occurrence time (for $t_{M}$ ). Its magnitude is given by $\hat{\omega}_{i_{M}}\left(t_{M}\right)$.

The proposed method can be summarised as in Figure 2.

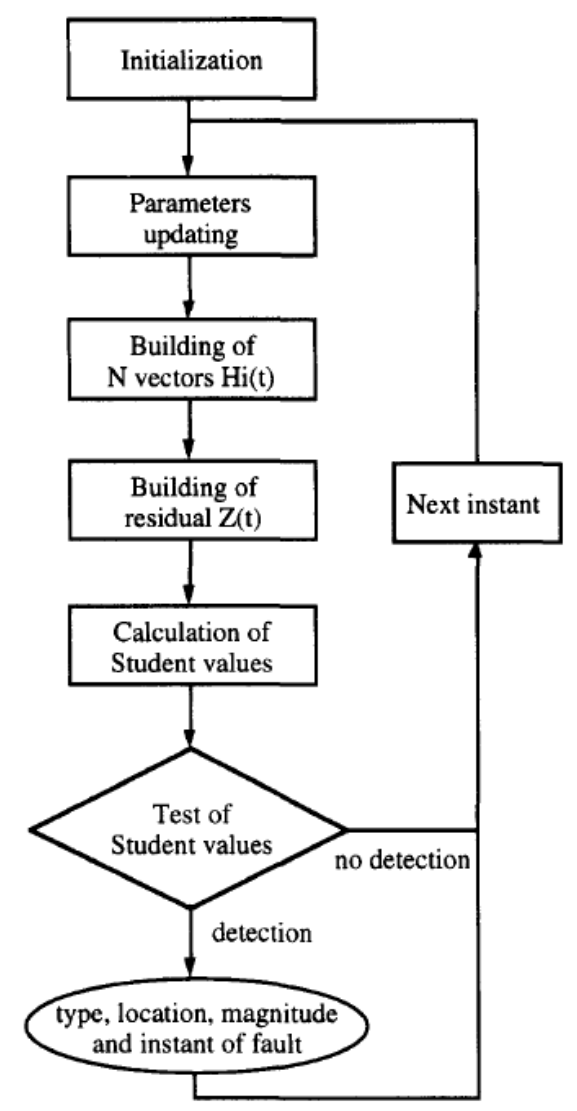

Figure 2: Complete procedure

\section{Application to the diagnosis of an annealing line}

The proposed method is applied to the monitoring of the strip drive in an n-roller system of a continuous annealing line. Others authors have previously dealt with the web tension modelling problem (Ouladsine et al., 1994).
The furnace composes the main device of the line. It gives the web a thermal cycle; each stage of the cycle corresponds to a metal transformation. The process consists of nine heating and cooling zones. It is separated from the entry section and the exit section by two looping towers. Because of these two looping towers, the operations performed on the strip in the entry section and the exit section can occur without disturbing the continuous flow of material in the centre. Figure 3 presents the

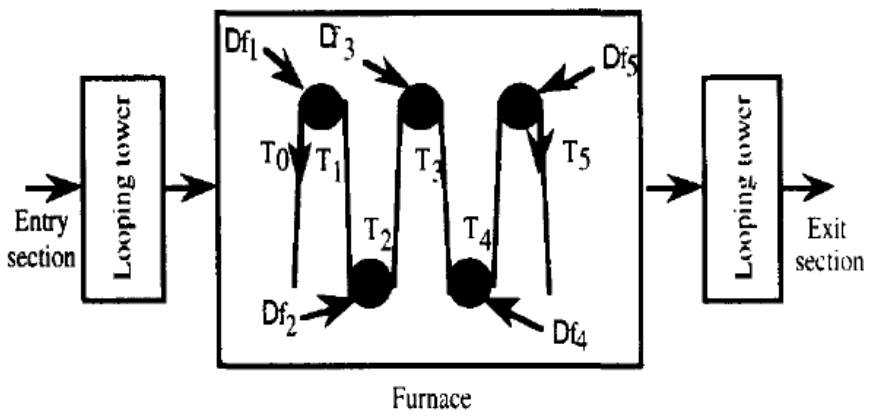

Figure 3: Continuous annealing line

strip drive in the furnace of the continuous annealing line where $T_{i}$ and $\Delta f_{i}$ are the web tension and the speed set point of drive motors respectively.

In order to identify the model of the tension behaviour in the furnace, a decomposition into smaller subsystems has been chosen (Parant et al., 1989). In this case, each subsystem corresponds to one output $T_{i}$. This approach presents experimental and numerical advantages: only the inputs of the subsystem under study have to be stimulated and the number of parameters to be estimated is not too large (Ouladsine et al., 1994).

Consider the subsystem of output $T_{2}$ (Figure 4 ). The corresponding model is chosen as a parametric discrete-time 4-input ARX model:

$A(q) T_{2}(t)=B_{1}(q) T_{1}(t)+B_{2}(q) T_{3}(t)+B_{3}(q) \Delta f_{1}(t)+B_{4}(q) \Delta f_{2}(t)$ with:

$$
\begin{aligned}
A(q) & =1+a_{1} q^{-1}+a_{2} q^{-2}, \\
B_{1}(q) & =b_{10}+b_{11} q^{-1}+b_{12} q^{-2}, \\
B_{2}(q) & =b_{20}+b_{21} q^{-1}, \\
B_{3}(q) & =b_{31} q^{-1}+b_{32} q^{-2}, \\
B_{4}(q) & =b_{41} q^{-1}+b_{42} q^{-2} .
\end{aligned}
$$

The model structure is obtained from an experimental study. To identify the system parameters, the input signals must sat-

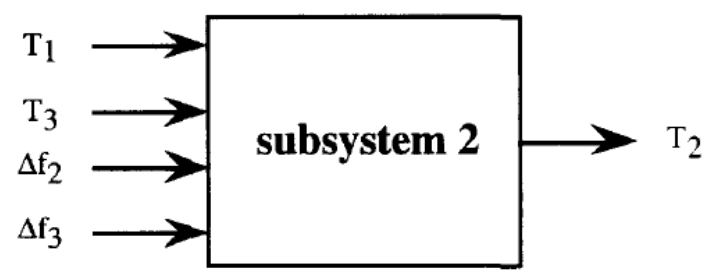

Figure 4: The subsystem of output $T_{2}$ 
isfy certain conditions. A minimal requirement is that all the system modes have to be persistently stimulated by the input signals over the measured period. To satisfy this condition, a Pseudo Random Binary Signal (PRBS) is used. In practice, a PRBS is a series of positive and negative steps around the mean value. The duration of the successive steps is chosen according to the spectrum band which corresponds to real dynamics of the system (Parant et al., 1989).

The measured inputs and output are considered without fault. Two experiments are carried out. In the first, a disturbance is added to the measured output at time point 350 . Figure 5

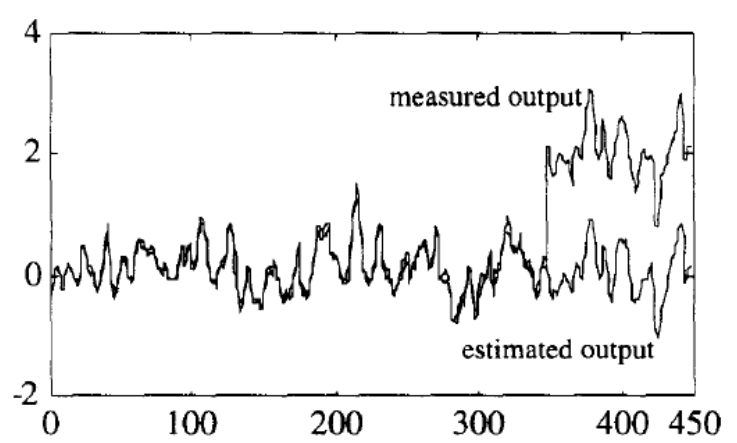

Figure 5: Measured and estimated system output

shows the disturbed measured output and the output estimated by the model with the parameters calculated by the robust online identification procedure. The disturbance function $f_{y}(t)$ represents a level change of magnitude $\omega=2$, and renders a bias of the sensor for $T_{2}$.

Figure 6 shows the evolution of some parameters estimated by the robust recursive identification procedure with the disturbed measurements (eq. 12 and 13). Notice that the estimated parameters are weakly influenced by the bias on output, beginning at time point 350 . The final values of the parameters are:

$$
\begin{aligned}
a_{1} & =0.9461, \quad a_{2}=-0.5540, \\
b_{10} & =0.8621, \quad b_{11}=-0.7189, \quad b_{12}=0.2314, \\
b_{20} & =0.3749, \quad b_{21}=-0.1535, \\
b_{31} & =-0.0120, \quad b_{32}=-0.0264, \\
b_{41} & =0.0110, \quad b_{42}=0.0229 .
\end{aligned}
$$

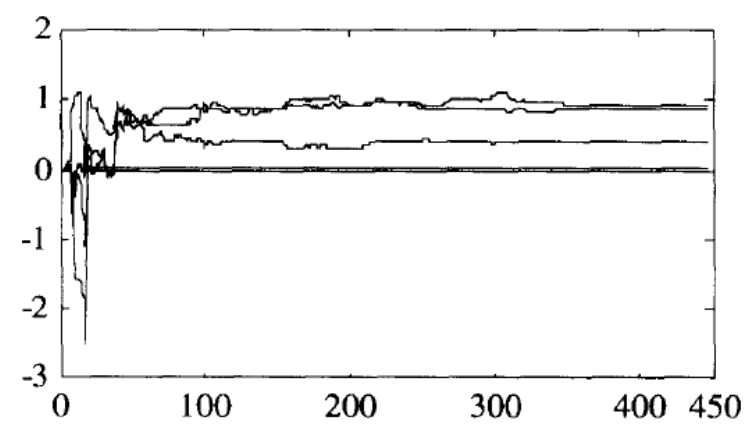

Figure 6: Parameters during recursive identification
Consequently, the disturbance acts on the prediction error sequence $z(t)$ calculated from the measurements, as shown in Figure 7.

It is supposed that $n_{D}=3$ shapes (impulse, step and drift) and

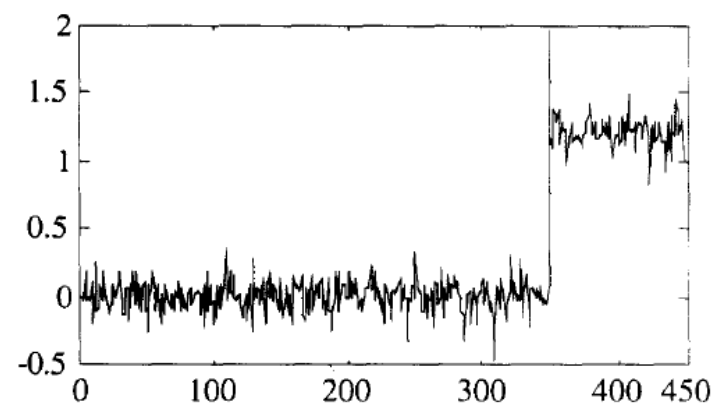

Figure 7: Residual $z(t)$

$n_{L}=5$ application points (inputs $T_{1}, T_{3}, \Delta f_{1}, \Delta f_{2}$ and output $T_{2}$ ) of a fault can be met ( $N=n_{D} n_{L}=15$ types of fault).

The previously described procedure is then carried out with a moving window of $n=2 m+1=21$ points. Figure 8 shows the maximum, for each moving window, of the $N$ variable $\left|t_{i}(t)\right|$ values (eq. 16).

Notice that a threshold strictly chosen as $t_{1-\alpha / 2} \approx 2$ with the

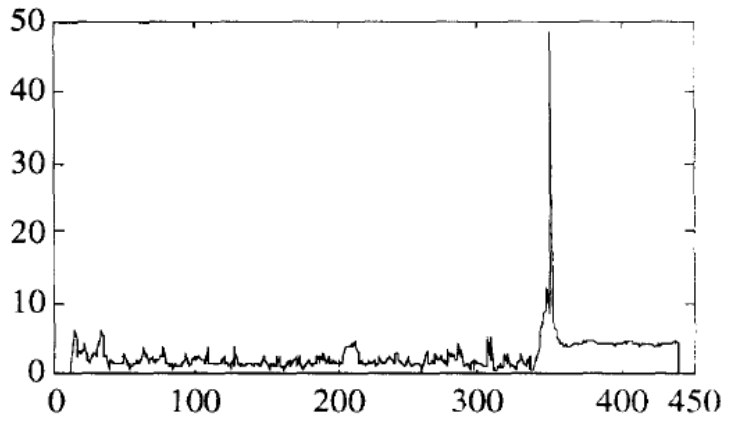

Figure 8: Maximum of $\left|t_{i}(t)\right|$ versus time

classic $\alpha=0.05$ and in this case $n-1=20$ degrees of freedom, can lead to many false alarms because the slightest disturbance in the residual can be explained by one of the different shapes $\underline{H}_{i}(t)$. Thus the threshold must be tuned to a higher value to provide the classical compromise between false alarms and misses. To illustrate the procedure, Figure 9 focuses on the closeness of the disturbance occurring at time 350 . The 15 variables $\left|t_{i}(t)\right|$, for $t=340, \ldots, 360$, are shown. Detection takes place at time point 347 , and the location and type determination of the fault are achieved exactly at time point 350 . The level change type and the location on $T_{2}$ are found. The corresponding estimation (eq. 14) of this level change is $\hat{\omega}=2.04(\omega=2)$.

Figure 10 shows the values of $Z(t)$ for the moving window centred at $t=350$, and the shapes $\underline{H}_{i}(t)$ corresponding to an outlier (impulse response) and to a bias (step response) on the output. Figure 10 highlights the basic principle of the procedure: to fit shapes on a moving window which characterise the different faults (type and location) on the transient period of the residual. 


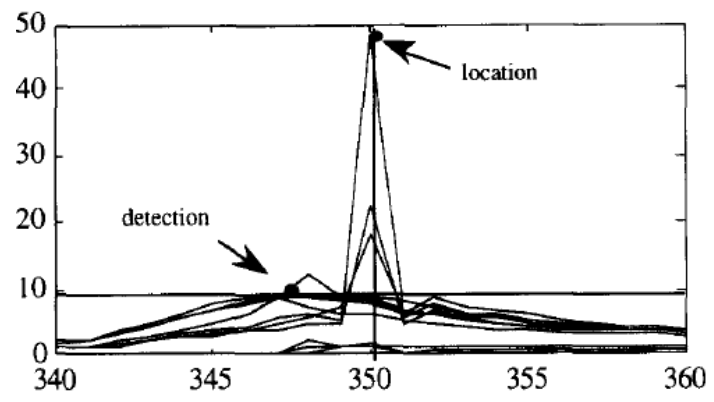

Figure 9: $\left|t_{i}(t)\right|, i=1, \ldots, 15$, versus time

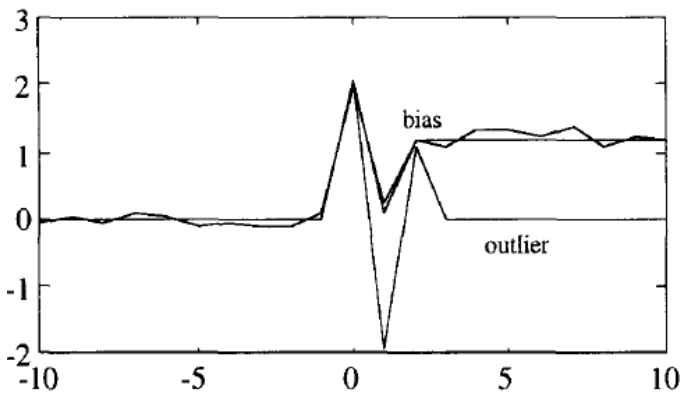

Figure 10: Residual and 2 shapes $\underline{H}_{i}(t)$

In the second experiment, a disturbance (a level change equal to 2 ) is added at time point 300 to the second input $T_{3}$. Figure 11 shows the resulting prediction error sequence $z(t)$, calculated as previously. This figure shows that the disturbance is not easily detectable.

Figure 12 presents the maximum, for each moving window, of

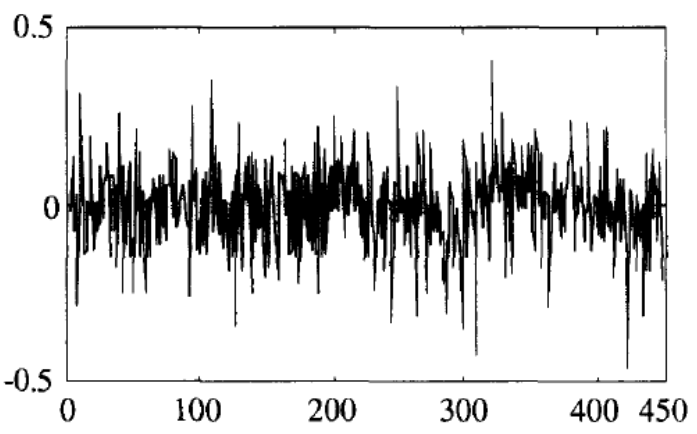

Figure 11: Residual $z(t)$

the $N$ variable $\left|t_{i}(t)\right|$ values (eq. 16). Notice that the proposed procedure has achieved the complete diagnosis of this disturbance and has given an estimation of the disturbance amplitude $\hat{\omega}=2.06$.

It would be interesting to compare the efficiency of other diagnostic methods applied to the system and the types of faults previously presented.

\section{Conclusion}

The proposed method appears attractive due to its simplicity and its unified character. The case of MIMO systems which can

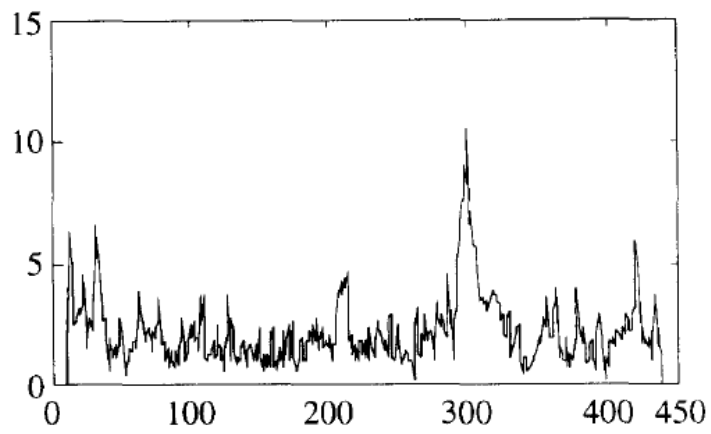

Figure 12: Maximum of $\left|t_{i}(t)\right|$ versus time

be broken up into several MISO systems leads to the implementation of as many complete diagnostic procedures as there are MIMO system outputs.

The robustness of the detection can be improved by using adaptive thresholds which are varied according to the level of the different $t_{i}$ variables on training periods without faults.

On the other hand, the proposed method is based on the discriminating character of the different shapes. Therefore if some polynomials of the model are close to each other, the isolation of the fault will be problematic.

When MIMO systems are considered, in the case of a fault on input, some redundancy occurs for the detection and isolation concerning the inputs involved in several subsystems. Therefore, the isolation power increases when some polynomials are close to each other in one particular subsystem.

It can be noted that because of the presence of the robust recursive parameter identification, the proposed method frees itself from slow non-stationarities of the system, which can be monitored from the estimated parameters. Extensions of the proposed method can be considered concerning, for example, the stochastic case, which leads to a state-space formulation. A robust Kalman filter can then be used.

\section{References}

Aström, K. J., 1980. Maximum likelihood and prediction error methods. Automatica $16(5), 551-574$.

Basseville, M., 1988. Detecting changes in signal and systems - A survey. Automatica 24 (3), 309-326.

Box, G. E. P., Tiao, G. C., 1975. Intervention analysis with applications to economic and environmental problems. J. Am. Stat. Assoc. 70 (349), 70 79.

Chen, J., Patton, R. J., 1994. A re-examination of fault detectability and isolability in linear dynamic systems. In: Proc. IFAC Symp. SAFEPROCESS'94. Espoo, Finland, June 3-16, pp. 590-596.

Frank, P. M., 1990. Fault diagnosis in dynamic systems using analytical and knowledge-based redundancy: A survey and some new results. Automatica 26 (3), 459-474.

Gomm, J. B., Williams, D., Harris, P., 1993. A generic method for fault detection in process control loops by recursive parameter estimation. European Journal of Diagnosis and Safety in Automation 3, 47-68.

Höfling, T., Pfeufer, T., 1994. Detection of additive and multiplicative faults - Parity space vs. parameter estimation. In: Proc. IFAC Symp. SAFEPROCESS'94. Espoo, Finland, June 3-16, pp. 539-544.

Huber, P. J., 1964. Robust estimation of a location parameter. Ann. Math. Stat. 35 (1), 73-101.

Isermann, R., 1984. Process fault detection based on modeling and estimation methods - A survey. Automatica 20 (4), 387-404. 
Isermann, R., 1994. Integration of fault detection and diagnosis methods. In: Proc. IFAC Symp. SAFEPROCESS'94. Espoo, Finland, June 3-16, pp. 597612.

Kobi, A., Ouladsine, M., Bloch, G., 1993a. Outliers detection, location and identification in time series. In: Proc. IMACS/IFAC Sec. Int. Symp. on Mathematical and Intelligent Models in System Simulation MIM-s ${ }^{2}$ '93. Brussels. Belgium, April 12-16, pp. 275-280.

Kobi, A., Ouladsine, M., Ragot, J., 1993b. Fault detection in ARX process. In: Proc. IEEE lnt. Conf. on Systems, Man and Cybernetics. Vol. 3. Le Touquet, France, October 17-20, pp. 394-399.

Masreliez, C. J., Martin, R. D., 1977. Robust bayesian estimation for the linear model and robustifying the Kalman filter. IEEE Trans. on Automatic Control 22 (3), 361-371.

Mironovski, L. A., 1980. Functional diagnosis of linear dynamic systems - A survey. Automation and Remote Control 41, 1122-1143.

Neumann, D., 1991. Fault diagnosis on machine tools by estimation of signal spectra. In: Proc. IFAC Symp. SAFEPROCESS'91. Vol. 1. Baden-Baden, Germany, September 10-13, pp. 73-78.

Ouladsine, M., Iung, C., Ragot, J., 1994. Recursive identification of interconnected systems. Application to the modelling of an annealing line. In: Proc. 1st IMACS Symp. on Mathematical Modelling, Mathmod Vienna. Vol. 2. Vienna, Austria, February 2-4, pp. 537-540.

Parant, F., Iung, C., Bello, P., 1989. Traction and speed control of an iron strip in a continuous annealing line. In: Proc. 3rd EPE Conference. October 9-11, pp. 1417-1419.

Patton, R. J., 1994. Robust model-based fault diagnosis: The state of the art. In: Proc. IFAC Symp. SAFEPROCESS'94. Vol. 1. Espoo, Finland, June 3-16, pp. 1-24.

Patton, R. J., Chen, J., 1991. A review of parity space approaches to fault diagnosis. In: Proc. IFAC Symp. SAFEPROCESS'91. Vol. 1. Baden-Baden, Germany, September 10-13, pp. 239-256.

Puthenpura, S., Sinha, N. K., 1990. A robust recursive identification method. Control Theory and Advanced Technology 6, 683-695.

Tsay, R. S., 1988. Outliers, level shifts, and variance changes in time series. J. of Forecasting 7 (1), 1-20.

Willsky, A. S., 1976. A survey of design methods for failure detection in dynamic systems. Automatica 12 (6), 601-611. 